\title{
SOME RESULTS ON SEMILINEAR SYSTEMS ON THE UNBOUNDED SPACE $\mathbb{R}^{3}$
}

\author{
J.M. MERCIER*
}

\begin{abstract}
We study in this paper the following systems, using standard tools devoted to the analysis of semilinear elliptic problems on $\mathbb{R}^{3}$ :

$R\left(\varepsilon_{1}, \varepsilon_{2}\right)\left\{\begin{array}{l}-\Delta u-\omega_{1}^{2} u=\varepsilon_{1} u v^{2} \\ -\Delta v-\omega_{2}^{2} v=\varepsilon_{2} v u^{2}\end{array}, \varepsilon_{i}= \pm 1, \omega_{i} \in \mathbb{R}\right.$, for $i=1,2$,

where $u, v$ holds for real valued functions. These systems do not admit any non trivial finite energy solution. However, we exhibit infinitely many non trivial radial solutions in the $\varepsilon_{1} \varepsilon_{2}=+1$ cases. A first type of solution consists in a ground state of $R(-1,-1)$, exhibited by variational arguments, whose structure is a finite energy perturbation of a non trivial constant solution of $R(-1,-1)$. A second type consists in a radial, oscillating, asymptotically null at infinity solution in the $\varepsilon_{1} \varepsilon_{2}=+1$ cases that we exhibit using eigenvalue comparison and ordinary differential equation type arguments.
\end{abstract}

\section{Introduction, statement of the results.}

In this paper, we look for particular standing wave solutions $(U, V)(t, x)=\left(e^{i \omega_{1} t} u(x), e^{i \omega_{2} t} v(x)\right)$, or particular travelling wave solutions $(U, V)(t, x)=(u(x-c t), v(x-c t)), c=\left(c_{1}, c_{2}, c_{3}\right) \in \mathbb{R}^{3},|c|<1$, of the following systems of wave equations

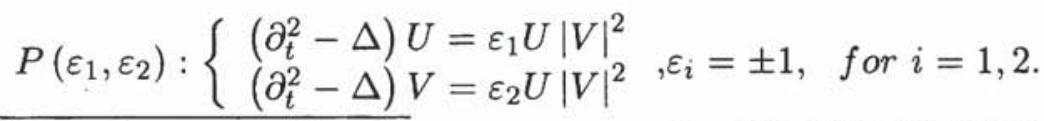

*This author was under European grant number ERBCHBGCT940607 and ERBFMRXCT960033 during the realisation of this paper.

1991 Mathematics Subject Classification: 65K10, 35J50, 35J45.

Servicio de Publicaciones. Universidad Complutense. Madrid, 2000 
The system $P(+1,-1)$ comes from field theory, (see [3], [5], [6] and references therein for a physical background), and we study the cases $\varepsilon_{1} \varepsilon_{2}=+1$ for a better understanding of this last case. It is straightforward to compute that a standing wave solution of $P\left(\varepsilon_{1}, \varepsilon_{2}\right)$ satisfies the system

$$
R\left(\varepsilon_{1}, \varepsilon_{2}\right)\left\{\begin{array}{l}
-\Delta u-\omega_{1}^{2} u=\varepsilon_{1} u v^{2} \\
-\Delta v-\omega_{2}^{2} v=\varepsilon_{2} v u^{2}
\end{array}, \varepsilon_{i}= \pm 1, \omega_{i} \in \mathbb{R}, \text { for } i=1,2,\right.
$$

A travelling wave solution satisfies also $R\left(\varepsilon_{1}, \varepsilon_{2}\right)$, but for $\omega_{1}=\omega_{2}=0$ and the change of variables

$$
\left\{\begin{array}{l}
u^{\prime}(x)=u\left(L^{1 / 2} x\right) \\
v^{\prime}(x)=v\left(L^{1 / 2} x\right)
\end{array} .\right.
$$

In the last expression, the symmetric positive definite matrix $L$ is given by $L=\left(L_{i j}\right)$, with $1 \leq i, j \leq 3$ and $L_{i j}=\delta_{i j}-c_{i} c_{j}$.

The systems $R\left(\varepsilon_{1}, \varepsilon_{2}\right)$ are semilinear elliptic systems on the unbounded space $\mathbb{R}^{3}$. It seems natural at a first sight to look for variational methods to exhibit non trivial solutions of $R\left(\varepsilon_{1}, \varepsilon_{2}\right)$, because they have a variational structure (see for instance [9] for a general introduction to variational methods) : each of the system $R\left(\varepsilon_{1}, \varepsilon_{2}\right)$ is obtained formally as the Euler-Lagrange equation of the following functional, or Lagrangian :

$$
\begin{aligned}
J_{\left(\varepsilon_{1}, \varepsilon_{2}\right)}(u, v)= & -\varepsilon_{1} \int_{\mathbf{R}^{3}}\left[(\nabla u)^{2}-\omega_{1}^{2} u^{2}\right] d x- \\
& -\varepsilon_{2} \int_{\mathbf{R}^{3}}\left[(\nabla v)^{2}-\omega_{2}^{2} v^{2}\right] d x+\int_{\mathbf{R}^{3}} u^{2} v^{2} d x
\end{aligned}
$$

Note that, if considered on a bounded domain $\Omega$ with Dirichlet conditions, the variational structure of $J_{\left(\varepsilon_{1}, \varepsilon_{2}\right)}(u, v)$ is of linking type. Thus, one can use the classical results of [8] or [11] to prove the existence of non trivial solutions whatever the signs of $\left(\varepsilon_{1}, \varepsilon_{2}\right)$ are for large enough $\Omega$. The same argumentation holds for non trivial periodical solutions in the monodimensional case. Thus in $\mathbb{R}^{3}$, there are infinitely many solutions that are periodic in one direction and constant in the two other orthogonal directions. For unbounded domains, particular solution of solitary wave type for semilinear Schrödinger, wave or heat equations leads often 
to consider variational problems. They have been widely studied for this purpose (see for instance [12], [4]). To study these functionals with a standard variational method, a natural functional setting is the energy space, i.e. the homogeneous Sobolev space made of square integrable first derivative functions

$$
W^{1}={\overline{\left\{\varphi \in C_{0}^{\infty}\left(\mathbb{R}^{3}\right)\right\}}}^{\|\nabla \varphi\|_{L^{2}(\mathrm{p} 3)},}
$$

where $C_{0}^{\infty}$ is the usual one of test functions. The semilinear part of the functionals $J_{\left(\varepsilon_{1}, \varepsilon_{2}\right)}$ are subcritical, respectively to the Sobolev critical exponent $2^{*}=6$ in $\mathbb{R}^{3}$. However, even in the cases $\varepsilon_{1} \varepsilon_{2}=+1$, they are strongly indefinite in the energy space. This is due to the "negative mass", i.e. the negative sign that appears on the $L^{2}$ norm in the expression of these. The linking structure still holds on the energy space, but a direct variational approach fails. Actually, the second section of this paper is devoted to the proof of the following non existence result of finite energy solution :

Proposition 1.1. It does not exist any non trivial finite energy solutions of $R\left(\varepsilon_{1}, \varepsilon_{2}\right)$ in the $\varepsilon_{1} \varepsilon_{2}=+1$ cases.

It does not exist any non trivial radial finite energy solutions of $R\left(\varepsilon_{1}, \varepsilon_{2}\right)$ in the $\varepsilon_{1} \varepsilon_{2}=-1$ cases.

We make a spherical symmetric assumption in the $\varepsilon_{1} \varepsilon_{2}=-1$ cases that we think to be only of technical nature.

This last result expresses the difficulty to find a good functional setting to study our systems via a variational method, that would be a good tool for the study of $R\left(\varepsilon_{1}, \varepsilon_{2}\right)$ in the $\varepsilon_{1} \varepsilon_{2}=-1$ cases. Actually it turns out in the third and fourth sections of this paper that the finite energy assumption is a too strong asymptotic behavior requirement.

A first idea to exhibit a non trivial solution is to perturb the systems $P\left(\varepsilon_{1}, \varepsilon_{2}\right)$ around a particular solution that is not of finite energy and to look for a better posed variational problem. This idea gives results perturbing $P(-1,-1)$ around its constant states. Doing so, one looks for a solution of the associated system of wave equations whose particular shape is $(U(t, x), V(t, x))=\left(a+u(x), e^{i \omega t} v(x)\right)$. We are led to consider the existence problem of a solution for

$$
R_{a}(-1,-1)\left\{\begin{array}{c}
-\Delta u=-(u+a) v^{2} \\
-\Delta v-\omega^{2} v=-v(u+a)^{2}
\end{array} .\right.
$$


If we look for finite energy solutions of this problem, we consider equivalently a critical point of the following Lagrangian

$$
J_{(-1,-1)}^{a}(u, v)=\int_{\mathbb{R}^{3}}(\nabla u)^{2} d x+\int_{\mathbb{R}^{3}}(\nabla v)^{2} d x+\int_{\mathbb{R}^{3}}\left((u+a)^{2} v^{2}-\omega^{2} v^{2}\right) d x .
$$

The quadratic part of this functional is now positive definite if $0<\omega^{2}<$ $a^{2}$, and we show in the third part of this paper that this functional has a mountain pass geometry. This allows us to prove the existence of a non trivial radial solution :

Theorem 1.1. For every $0<\omega^{2}<a^{2}$ there exists a non trivial radial solution $\left(u_{1}, v_{1}\right)$ of $R_{a}(-1,-1)$ verifying

$$
\sup _{(u, v) \in W^{1}, \quad(u, v) \neq 0} \frac{\int_{\mathbb{R}^{3}}\left[\omega^{2} v^{2}-(u+a)^{2} v^{2}\right] d x}{\left(\int_{\mathbb{R}^{3}}(\nabla u)^{2} d x\right)^{2} \int_{\mathbb{R}^{3}}(\nabla v)^{2} d x}=\frac{1}{\left(\int_{\mathbb{R}^{3}}\left(\nabla u_{1}\right)^{2} d x\right)^{2}} .
$$

This last equality can be viewed as a Sobolev type inequality. We show also that $\left(u_{1}, v_{1}\right)$ realizes the minimum of $J_{(-1,-1)}^{a}(u, v)$ among all the non trivial finite energy solutions of $R_{a}(-1,-1)$, defining a ground state type solution. So we expect that the corresponding solutions of $P(-1,-1)$ associated to these ground states be stable.

So far we have exhibited some solutions that are asymptotically constant at infinity, with a non null constant $(0, a)$. But we expect, due to the linking structure of our problems, to find non trivial solutions that are asymptotically null at infinity. We get two results in this direction, studying the equivalent systems of ordinary differential equations satisfied by any smooth radial solution of $R\left(\varepsilon_{1}, \varepsilon_{2}\right)$. These results may be known since we use very classical tools to prove them, but as we don't know a reference, we give a quick proof of them in the fourth part of this paper. Considering first $R(+1,+1)$, we prove easily (we denote $r(x)=\left(x_{1}^{2}+x_{2}^{2}+x_{3}^{2}\right)^{1 / 2}$ the radial coordinate) :

Theorem 1.2. For every $(a, b) \in \mathbb{R}^{2}$ there exists a radial solution $(u, v)$ of $R(+1,+1)$

verifying $\{u(0)=a, v(0)=b\}$ and $\left(\omega_{1}^{2} u^{2}+\omega_{2}^{2} v^{2}\right)(x) \leq \frac{C}{r^{2}(x)}$.

These solutions expressed in polar coordinates are oscillating ones in the $\omega_{1} \omega_{2} \neq 0$ and $a b \neq 0$ cases. To be more precise, let us (when properly 
defined) denote $\left\{\zeta_{i}(u)\right\}_{i \in N}$ the increasing sequence defined by the zeros of a smooth function $u$, then these solutions satisfy $\left|\zeta_{i+1}(u)-\zeta_{i}(u)\right| \rightarrow$ $\frac{\pi}{\left|\omega_{1}\right|},\left|\zeta_{i+1}(v)-\zeta_{i}(v)\right| \rightarrow \frac{\pi}{\left|\omega_{2}\right|}$, as $i \rightarrow \infty$. Considering now $R(-1,-1)$, we get a partial result, studying a particular case that we denote

$$
R(-1):-\Delta u-\omega^{2} u=-u^{3} .
$$

For this equation, we prove

Theorem 1.3. For every $\omega^{2}>a^{2}>0$, there exists a radial solution $u$ of $R(-1)$ verifying $\{u(0)=a\}, \lim \sup _{r(x) \rightarrow \infty} r^{2}(x)\left(\omega_{1}^{2} u^{2}+\omega_{2}^{2} v^{2}\right)(x)>$ 0 and $\lim _{r(x) \rightarrow \infty} u(x)=0$.

This is a sharp existence result in the sense that the solution of the ordinary differential equation associated to $R(-1)$ is $u=\omega$ for initial conditions $\left\{u(0)^{2}=\omega^{2}, \partial_{r} u(0)=0\right\}$, and that this equation blows up solution within finite time when it is associated to initial conditions satisfying $\left\{u(0)^{2}>\omega^{2}, \partial_{r} u(0)=0\right\}$. Here also we have the property $\left|\zeta_{i+1}(u)-\zeta_{i}(u)\right| \rightarrow \frac{\pi}{|\omega|}, i \rightarrow \infty$.

Remark 1.1. Let us consider the semilinear Klein Gordon equation $\left\{\partial_{t}^{2} U-\Delta U+m^{2} U=-|U|^{2} U\right\}$ in the three dimensional case. It is a well known fact that there exists a stable solitary wave type solution of this equation whose shape is $U(t, x)=e^{i w t} u(x)$ in the cases $0 \leq \omega^{2} \leq$ $m^{2}$ (see [12], [4]). It is interesting to note that for higher frequencies, i.e. in the case $0<m^{2}<\omega^{2}$, there exists still solitary wave that are asymptotically null at infinity (theorem 1.3), but that are no longer of finite energy (proposition 1.1). An interesting question is whether these solutions are stable or not.

Notations. The usual Sobolev space over $\mathbb{R}^{3}$ is denoted $H^{1}$. The space $W_{r}^{1}$ (resp. $H_{r}^{1}$ ) denotes the closed subset of $W^{1}$ (resp. $H^{1}$ ) of radial functions. For a function $u, \operatorname{supp} u$ holds for its support. We quote the multivalued function $\operatorname{sgn}(y)=\{-1, y \leq 0 ;+1 ; y \geq 0\}$. The annulus $\{x ; a \leq r(x) \leq b\}, 0<a$, is $B(a, b)$. The measure of the unit ball is $\alpha_{3}=$ meas $\{B(0,1)\}$. We recall the usual Sobolev embedding in $\mathbb{R}^{3}$ :

$$
W^{1}\left(\mathbb{R}^{3}\right) \hookrightarrow L^{6}\left(\mathbb{R}^{3}\right), \quad H^{1}\left(\mathbb{R}^{3}\right) \hookrightarrow L^{p}\left(\mathbb{R}^{3}\right), 2 \leq p \leq 6 .
$$




\section{Non existence result in finite energy spaces}

As a first step to prove proposition (1.1), we first get some a priori informations that verify a finite energy solution of $R\left(\varepsilon_{1}, \varepsilon_{2}\right)$ :

Proposition 2.1. Every finite energy $(u, v)$ solution of $R\left(\varepsilon_{1}, \varepsilon_{2}\right)$ is $C^{\infty}$ and verifies

i) (energy type relations)

$$
\int_{\mathbb{R}^{3}}(\nabla u)^{2}-\omega_{1}^{2} \int_{\mathbb{R}^{3}} u^{2}=\varepsilon_{1} \int_{\mathbb{R}^{3}} u^{2} v^{2} ; \int_{\mathbb{R}^{3}}(\nabla v)^{2}-\omega_{2}^{2} \int_{\mathbb{R}^{3}} u^{2}=\varepsilon_{2} \int_{\mathbb{R}^{3}} u^{2} v^{2}
$$

ii) (Pohozaev type relations)

$$
\int_{\mathbb{R}^{3}} \varepsilon_{1}(\nabla u)^{2}+\varepsilon_{2}(\nabla v)^{2}=3\left(\int_{\mathbb{R}^{3}} u^{2} v^{2}+\varepsilon_{1} \omega_{1}^{2} \int_{\mathbb{R}^{3}} u^{2}+\varepsilon_{2} \omega_{2}^{2} \int_{\mathbb{R}^{3}} v^{2}\right)
$$

This proposition uses a classical argumentation. We present a quick proof to the reader because much care has to be taken to handle integrations by parts that are in the heart of the proof. As an example, these integrations fails proving the first energy relation related to the quite similar system $R_{a}(-1,-1)$ (see next section).

\section{Proof.}

proof of $i$ ) The idea is to use the multiplier $(u, v)$ and to integrate by parts. The only technical point is to justify these integrations for an a priori finite energy solution $(u, v) \in W^{1}$ of $R\left(\varepsilon_{1}, \varepsilon_{2}\right)$. For this purpose, we introduce the following truncature function of the unit ball,

$$
\begin{gathered}
\chi_{B(0,1)}(x)=1, \text { if } x \in B(0,1) \\
\chi_{B(0,1)}(x)=0, \text { if } x \in \mathbb{R}^{3} \backslash B(0,2) ; \\
0 \leq \chi_{B(0,1)}(x) \leq 1,
\end{gathered}
$$

and $\chi_{B(0,1)} \in C_{0}^{\infty}$. We introduce also for short $\chi_{k}(x)=\chi_{B(0,1)}\left(\frac{x}{k}\right)$, $u_{k}=\chi_{k} u, v_{k}=\chi_{k} v$. We have the property $0 \leq u_{k} u \leq u^{2}, 0 \leq v_{k} v \leq v^{2}$ because of the choice of the truncated function.

Using the Poincare inequality, $\left(u_{k}, v_{k}\right)$ belongs to $H^{1}$. As we deal here with a subcritical problem, the $C^{\infty}$ property of the solution is easy to prove via a classical bootstrap argument. As a consequence, we have also that every finite energy solution is uniformly bounded and 


$$
\lim _{r(x) \rightarrow \infty}(|u(x)|+|v(x)|)=0 .
$$

We multiply the system $R\left(\varepsilon_{1}, \varepsilon_{2}\right)$ with $\left(u_{k}, v_{k}\right)$, and integrate over $\mathbb{R}^{3}$ in order to get

$$
\left\{\begin{array}{c}
\int_{\mathbb{R}^{3}} \nabla u \nabla u_{k}=\varepsilon_{1} \int_{\mathbb{R}^{3}} u u_{k} v^{2}+\omega_{1}^{2} \int_{\mathbb{R}^{3}} u u_{k} \\
\int_{\mathbb{R}^{3}} \nabla v \nabla v_{k}=\varepsilon_{2} \int_{\mathbb{R}^{3}} u^{2} v v_{k}+\omega_{2}^{2} \int_{\mathbb{R}^{3}} v v_{k}
\end{array}\right.
$$

We estimate with the Hölder inequality

$$
\begin{aligned}
\int_{\mathbb{R}^{3}} u u_{k} v^{2} & \leq C\left\|u u_{k}\right\|_{L^{3 / 2}}\left\|v^{2}\right\|_{L^{3}} \leq C\left\|u u_{k}\right\|_{L^{3 / 2}}\left\|v^{2}\right\|_{L^{3}} \leq \\
& \leq C\left\|u u_{k}\right\|_{L^{\infty}}^{1 / 3}\left\|u u_{k}\right\|_{L^{1}}^{2 / 3}\|v\|_{L^{6}}^{2}
\end{aligned}
$$

So we have $\int_{\mathbb{R}^{3}} u u_{k} v^{2} \leq C\left(\int_{\mathbb{R}^{3}} u u_{k}\right)^{2 / 3}$, using (2) and (3). In the same way $\int_{\mathbb{R}^{3}} v v_{k} u^{2} \leq\left(\int_{\mathbb{R}^{3}} v v_{k}\right)^{2 / 3}$.

We recall that $\left(u_{k}, v_{k}\right) \rightarrow(u, v)$ in $W^{1}$, as $k \rightarrow \infty$, according to ([2], p.230). Thus the left hand side of (4) is uniformly bounded. Using the previous estimates, we get

$$
\left\{\begin{array}{l}
\infty>\omega_{1}^{2} \int_{\mathbb{R}^{3}} u u_{k}-C\left(\int_{\mathbb{R}^{3}} u u_{k}\right)^{2 / 3} \\
\infty>\omega_{2}^{2} \int_{\mathbb{R}^{3}} v v_{k}-C\left(\int_{\mathbb{R}^{3}} v v_{k}\right)^{2 / 3}
\end{array} .\right.
$$

Thus $\left(\omega_{1}^{2} \int_{\mathbb{R}^{3}} u u_{k}\right)_{k \geq 0}$ and $\left(\omega_{2}^{2} \int_{\mathbb{R}^{3}} v v_{k}\right)_{k \geq 0}$ are positive, increasing, uniformly bounded sequences. Using the Beppo-Levi theorem we have

$$
\left\{\begin{array}{l}
\varepsilon_{1} \int_{\mathbb{R}^{3}} u u_{k} v^{2} \rightarrow \int_{\mathbb{R}^{3}}(\nabla u)^{2}-\omega_{1}^{2} \int_{\mathbb{R}^{3}} u^{2}=\varepsilon_{1} \int_{\mathbb{R}^{3}} u^{2} v^{2}<\infty \\
\varepsilon_{2} \int_{\mathbb{R}^{3}} v v_{k} u^{2} \rightarrow \int_{\mathbb{R}^{3}}(\nabla v)^{2}-\omega_{2}^{2} \int_{\mathbb{R}^{3}} v^{2}=\varepsilon_{2} \int_{\mathbb{R}^{3}} u^{2} v^{2}<\infty
\end{array},\right.
$$

that ends the proof of part i).

proof of ii) We note here $\partial_{x_{i}} u=u_{i}, \partial_{r} u=\left(\partial_{x_{1}} u, \partial_{x_{2}} u, \partial_{x_{3}} u\right)$, $n=\left(n_{1}, n_{2}, n_{3}\right)$ the normal outward of the sphere of radius $r$, denoted $\partial B_{r}$, and $d S$ the measure over $\partial B_{r}$. We use the Einstein's summation convention and follow the proof given in ([4]).

We multiply $R\left(\varepsilon_{1}, \varepsilon_{2}\right)$ with the usual multiplier for Pohozaev identity that is $\left(\varepsilon_{1} u_{i} x_{i}, \varepsilon_{2} v_{i} x_{i}\right)$, and integrate over a ball $B(0, r)$. We consider first the left hand integral 


$$
I_{r}=-\varepsilon_{1} \int_{B(0, r)}\left(v_{j, j}\right) v_{i} x_{i}-\varepsilon_{2} \int_{B(0, r)}\left(u_{j, j}\right) u_{i} x_{i}
$$

We have (and its equivalent for $u$ )

$$
\int_{B(0, r)}\left(v_{j, j}\right) v_{i} x_{i}=\frac{1}{2}\left(\int_{B(0, r)}(\nabla v)^{2}+\int_{\partial B_{r}} r\left(\partial_{r} v\right)^{2} d S .\right)
$$

On the right hand, we calculate

$$
\begin{aligned}
I_{r} & =\int_{B(0, r)}\left(v^{2}+\varepsilon_{1} \omega_{1}^{2}\right) u u_{i} x_{i}+\left(u^{2}+\varepsilon_{2} \omega_{2}^{2}\right) v v_{i} x_{i}= \\
& =\frac{1}{2} \int_{B(0, r)} \partial_{i}\left(u^{2} v^{2}+\varepsilon_{1} \omega_{1}^{2} u^{2}+\varepsilon_{2} \omega_{2}^{2} v^{2}\right) x_{i}= \\
& =-\frac{3}{2} \int_{B(0, r)} u^{2} v^{2}+\varepsilon_{1} \omega_{1}^{2} u^{2}+\varepsilon_{2} \omega_{2}^{2} v^{2}+ \\
& +\frac{1}{2} \int_{\partial B_{r}}\left[u^{2} v^{2}+\varepsilon_{1} \omega_{1}^{2} u^{2}+\varepsilon_{2} \omega_{2}^{2} v^{2}\right] r d S
\end{aligned}
$$

Thus we compute

$$
\begin{aligned}
& \int_{B(0, r)} \varepsilon_{1}(\nabla u)^{2}+\varepsilon_{2}(\nabla v)^{2}= \\
= & -3\left(\int_{B(0, r)} u^{2} v^{2}+\varepsilon_{1} \omega_{1}^{2} \int_{B(0, r)} u^{2}+\varepsilon_{2} \omega_{2}^{2} \int_{B(0, r)} v^{2}\right)+C(r)
\end{aligned}
$$

with

$$
C(r)=\frac{1}{2} \int_{\partial B_{r}} r\left(u^{2} v^{2}+\varepsilon_{1} \omega_{1}^{2} u^{2}+\varepsilon_{2} \omega_{2}^{2} v^{2}+\varepsilon_{1}\left(\partial_{r} u\right)^{2}+\varepsilon_{2}\left(\partial_{r} v\right)^{2}\right) d S .
$$

We use here the finite energy hypothesis, noting that, according to the part i) of Proposition, the function $f=u^{2} v^{2}+\varepsilon_{1} \omega_{1}^{2} u^{2}+\varepsilon_{2} \omega_{2}^{2} v^{2}+$ $\varepsilon_{1}\left(\partial_{r} u\right)^{2}+\varepsilon_{2}\left(\partial_{r} v\right)^{2}$ belongs to $L^{1}\left(\mathbb{R}^{3}\right)$. Thus we deduce

$$
\left|\int_{0}^{\infty} \frac{C(r)}{r} d r\right|=\left|\int_{0}^{\infty} \int_{\partial B_{r}} f d S d r\right|=\|f\|_{L^{1}}<\infty .
$$


implying the existence of a subsequence $\left(r_{n}\right)_{n \in \mathbb{N}}$ that tends to infinity and for which $C\left(r_{n}\right) \rightarrow 0, n \rightarrow \infty$. We estimate $I_{r}$ over such a subsequence in order to end the proof of Proposition.

Proof of Proposition 1.1. We consider two cases

$\varepsilon_{1}=\varepsilon_{2}:$ we denote for short the functionals $x=\int_{\mathbf{R}^{3}}(\nabla u)^{2}+$ $\int_{\mathbf{R}^{3}}(\nabla v)^{2}, y=\omega_{1}^{2} \int_{\mathbf{R}^{3}} u^{2}+\omega_{2}^{2} \int_{\mathbf{R}^{3}} v^{2}, z=\int_{\mathbf{R}^{3}} u^{2} v^{2}$. Using Proposition 2.1 , we get the following system

$$
\left\{\begin{array}{c}
x=2 \varepsilon_{1} z+y \\
x=3\left(\varepsilon_{1} z+y\right)
\end{array}\right.
$$

implying that $x=-3 y \leq 0$. Thus $(u, v)$ is the trivial solution.

$\varepsilon_{1}=-\varepsilon_{2}:$ the proof relies on an indirect argument. We consider $\left(\varepsilon_{1}, \varepsilon_{2}\right)=(+1,-1)$, the other case $\left(\varepsilon_{1}, \varepsilon_{2}\right)=(-1,+1)$ being symmetric. There is an alternative :

First case. $\omega_{2}=0$. Proposition 2.1 i) implies $\int_{\mathbf{R}^{3}} u^{2} v^{2}=-\int_{\mathbf{R}^{3}}(\nabla v)^{2}=$ 0 . If $\omega_{1}=0$, we deduce $\int_{\mathbf{R}^{3}}(\nabla u)^{2}=0$, thus $(u, v)$ is the trivial solution. If $\omega_{1} \neq 0$ then $u \in L^{2}$, according to Proposition 2.1 i). Furthermore $u$ satisfies $-\Delta u-\omega_{1}^{2} u=0$, thus $u=0$ because the only finite energy solution of this problem is the trivial one.

Second case. $\omega_{2} \neq 0$. In this case, we suppose that $(u, v) \in W_{r}^{1}$. We deduce $v \in L^{2}$ from Proposition $2.1 \mathrm{i}$ ). Furthermore, $v$ satisfies the equation $-\Delta v+u^{2} v=\omega_{2}^{2} v$. We recall the following result of Kato, ([10]),

The operator $(-\Delta+q(x))$ does not admit any positive eigenvalue on $L^{2}\left(\mathbf{R}^{3}\right)$ under the hypothesis

$$
q(x) \text { is a continuous function outside of a compact set, }
$$

$$
\lim _{r(x) \rightarrow \infty} r(x) q(x)=0 .
$$

To use this result, we precise the asymptotic behavior of a finite energy radial function in the following lemma :

Lemma 2.1. Let $u \in W_{r}^{1}$. Then $u$ is equal outside of the origin to a continuous function $u_{s}(r(x))$ whose asymptotic behavior is $\lim _{r \rightarrow \infty} r^{1 / 2} u_{s}(r)=0$, and $\lim _{r \rightarrow 0} r^{1 / 2} u_{s}(r)=0$. 
Proof. Let $u \in W_{r}^{1}$ and $u_{s}(r(x))=u(x)$. Notice that we have, using spherical coordinates

$$
\left\|\nabla u ; L^{2}\left(\mathbf{R}_{x}^{3}\right)\right\|^{2}=\alpha_{3}\left\|r \partial_{r} u_{s} ; L^{2}\left(\mathbf{R}_{r}^{+}\right)\right\|^{2}<\infty .
$$

Notice too that (2) implies

$$
\left\|r^{2} u_{s}^{6} ; L^{1}\left(\mathbf{R}_{r}^{+}\right)\right\|=\left\|u ; L^{6}\left(\mathbf{R}_{x}^{3}\right)\right\|^{6} \leq C\left\|\nabla u ; L^{2}\left(\mathbf{R}_{x}^{3}\right)\right\|^{6}<\infty .
$$

Using the Sobolev injections, $u_{s}$ is a continuous function over $\left.] 0,+\infty\right]$. So we can compute the following integration by parts :

$\infty>\left\|r \partial_{r} u_{s} ; L^{2}([1, a])\right\|^{2}=\left\|\partial_{r}\left(r u_{s}\right) ; L^{2}([1, a])\right\|^{2}-\left[r u_{s}^{2}\right]_{1}^{a}$, for $a>0$.

Let $a \rightarrow \infty$. As $\left\|\partial_{r}\left(r u_{s}\right) ; L^{2}([1, a])\right\|^{2}$ is an increasing functions of $a$, we deduce $\lim _{a \rightarrow \infty} a u_{s}^{2}(a)=l_{\infty} \leq \infty$. Using (8) it turns out that $l_{\infty}=0$. In the same vein we have $\lim _{a \rightarrow 0} a u_{s}^{2}(a)=l_{0} \leq \infty$, so $l_{0}=0$ as well.

We deduce from this lemma $\lim _{r(x) \rightarrow \infty} r(x) u^{2}(x)=0$. This means according to Kato's result that $v \equiv 0$, and we deduce $u \equiv 0$, ending the proof of Proposition 2.1.

\section{Existence result using perturbative variational method}

In this part we consider the system

$$
R_{a}(-1,-1)\left\{\begin{array}{c}
-\Delta u=-(u+a) v^{2} \\
-\Delta v-\omega^{2} v=-v(u+a)^{2}
\end{array} .\right.
$$

We define the functionals

$$
I(u, v)=\int_{\mathbf{R}^{3}}(u+a)^{2} v^{2} d x-\omega^{2} \int_{\mathbf{R}^{3}} v^{2} d x ; J(u)=\int_{\mathbf{R}^{3}}(\nabla u)^{2} d x .
$$

We have for instance $J_{(-1,-1)}^{a}(u, v)=J(u)+J(v)+I(u, v)$. We first state some a priori relations that are satisfied by any finite energy solution $(u, v)$ of $R_{a}(-1,-1)$. This is a problem with a subcritical Sobolev 
exponent, so $(u, v)$ are $C^{\infty}$. Multiplying the second equation with $v$ and integrating by parts we get an energy type relation following the proof of proposition $2.1 \mathrm{i}$ )

$$
J(v)=-I(u, v) .
$$

Also, using the multiplier $\left(\left(u_{i}+a\right) x_{i}, v_{i} x_{i}\right)$ over $R_{a}(-1,-1)$ we get a Pohozaev type relation, following Proposition 2.1 ii)

$$
J(u)+J(v)=-3 I(u, v) .
$$

We deduce from these two relations that every finite energy solutions of $R_{a}(-1,-1)$ satisfies

$$
J_{(-1,-1)}^{a}(u, v)=J(u) ; \quad J(v)=2 J(u) .
$$

Remark 3.1. It is not possible to state an energy type relation using the first equation of $R_{a}(-1,-1)$. This is related to the fact that we deal with a "zero mass" case for $u$. Thus it is hopeless to look for a non trivial solution $u$ of $R_{a}(-1,-1)$ that belongs to $H^{1}$ : using the multiplier $\chi_{k}(u+a)$ (the notations used here are defined in the proposition $2.1 \mathrm{i}$ ) ) on the first equation of $R_{a}(-1,-1)$ and integrating by parts we get

$$
\int_{\mathbf{R}^{3}} \nabla u \nabla u_{k}+\int_{\mathbf{R}^{3}} \chi_{k}(u+a)^{2} v^{2}=a \int_{\mathbf{R}^{3}}\left(\Delta \chi_{k}\right) u
$$

We estimate

$$
\int_{\mathbf{R}^{3}}\left(\Delta \chi_{k}\right) u \leq\|u\|_{L^{2}(B(0,2 k))}\left\|\Delta \chi_{k}\right\|_{L^{2}}=C k^{-1 / 2}\|u\|_{L^{2}(B(0,2 k))} .
$$

If we suppose $u \in H^{1}$, then $\int_{\mathbf{R}^{3}}\left(\Delta \chi_{k}\right) u \rightarrow 0, k \rightarrow \infty$, and from (10) we deduce $u \equiv 0$.

To prove the existence of a non trivial solution for $R_{a}(-1,-1)$, we study a constrained minimization problem and we use Lagrange multipliers. We define the subset over the space $W^{1} \times H^{1}$,

$$
S_{\lambda, \mu}=\{J(u)=\lambda ; \quad J(v)=\mu\} .
$$

This subset is the constraint of our minimization problem that we express in the following way 


$$
K(\lambda, \mu)=\inf _{(u, v) \in S_{\lambda, \mu}} I(u, v) .
$$

We denote $S_{\lambda, \mu}^{r}$ the subset of $S_{\lambda, \mu}$ made of radially symmetric functions. The essential properties of this problem are quoted in the following proposition (we give the proof of the intermediate results at the end of this section).

Proposition 3.1. Let $0<\omega^{2}<a^{2}$. Then

i) $-\infty<K(\lambda, \mu)<0$.

ii) $K(\lambda, \mu)=K(1,1) \lambda^{2} \mu$.

iii) $K(\lambda, \mu)=\inf _{(u, v) \in S_{\lambda, \mu}^{r}} I(u, v)$

So, we can define a radial minimizing sequence that achieves $K(\lambda, \mu)$, that is a sequence $\left(u_{\lambda}^{n}, v_{\mu}^{n}\right)_{n \geq 0}$ that satisfies

$$
\left(u_{\lambda}^{n}, v_{\mu}^{n}\right) \in S_{\lambda, \mu}^{r}, I\left(u_{\lambda}^{n}, v_{\mu}^{n}\right) \rightarrow K(\lambda, \mu) \text {, as } n \rightarrow \infty .
$$

The main result concerning $K(\lambda, \mu)$ is :

Lemma 3.1. Let $0<\omega^{2}<a^{2}$. Then every radial symmetric minimizing sequence achieving $K(\lambda, \mu)$ admits a strongly convergent subsequence in $W_{r}^{1} \times H_{r}^{1}$.

Thus, considering $\left(u_{\lambda}^{n}, v_{\mu}^{n}\right)_{n \geq 0}$ a minimizing sequence, we can extract a subsequence converging to a function in $W_{r}^{1} \times H_{r}^{1}$ that we denote by $\left(u_{\lambda}, v_{\mu}\right)$. So there exists two Lagrange multipliers $\left(\theta_{\lambda}, \theta_{\mu}\right)$ such that $\left(u_{\lambda}, v_{\mu}\right)$ satisfies the following system :

$$
\left\{\begin{array}{c}
\theta_{\lambda}\left(-\Delta u_{\lambda}\right)=\left(u_{\lambda}+a\right) v_{\mu}^{2} \\
\theta_{\mu}\left(-\Delta v_{\mu}\right)=v_{\mu}\left(u_{\lambda}+a\right)^{2}-\omega^{2} v_{\mu}
\end{array} .\right.
$$

The energy relation for the second equation is $\theta_{\mu} J\left(v_{\mu}\right)=I\left(u_{\lambda}, v_{\mu}\right)$. The Pohozaev relation for this system is $\theta_{\lambda} \int_{\mathbf{R}^{3}}\left(\nabla u_{\lambda}\right)^{2}+\theta_{\mu} \int_{\mathbf{R}^{3}}\left(\nabla v_{\mu}\right)^{2}=$ $3 I\left(u_{\lambda}, v_{\mu}\right)$. So we deduce the signs of $\theta_{\mu}$ and $\theta_{\lambda}$ from Proposition 3.1, ii) :

$$
\theta_{\lambda}=2 K(1,1) \lambda \mu<0, \theta_{\mu}=K(1,1) \lambda^{2}<0 .
$$

Notice that $\left(u_{\lambda}, v_{\mu}\right)$ provides a solution of $R_{a}(-1,-1)$ using the rescaling $\left(u_{1}, v_{1}\right)=T_{\left(\left|\theta_{\lambda}\right|,\left|\theta_{\mu}\right|\right)}\left(u_{\lambda}, v_{\mu}\right)$ defined by 


$$
\left(u^{a}, v^{b}\right)=T_{(a, b)}(u, v), \quad \text { with } \quad\left\{\begin{array}{c}
u^{a}(x)=u(x \sqrt{b}) \\
v^{b}(x)=\sqrt{\frac{b}{a}} v(x \sqrt{b})
\end{array}\right.
$$

where $(\alpha, \beta)$ are two strictly positive constants. The properties of this rescaling are

$$
J\left(u^{a}\right)=\frac{1}{\sqrt{b}} J(u) ; J\left(v^{b}\right)=\frac{\sqrt{b}}{a} J(v) ; I\left(u^{a}, v^{b}\right)=\frac{1}{a \sqrt{b}} I(u, v) .
$$

We compute that our solution satisfies $J_{(-1,-1)}^{a}\left(u_{1}, v_{1}\right)=J\left(u_{1}\right)=$ $\frac{1}{\sqrt{|K(1,1)|}}$. Note that every finite energy solution $(u, v)$ of $R_{a}(-1,-1)$ satisfies, according to (9) and Proposition 3.1, ii),

$2 J(u)=J(v)=-I(u, v) \leq-K(1,1) J(u)^{2} J(v) \Rightarrow J(u)+K(1,1) J(u)^{3} \leq 0$.

We deduce from this last relation $J_{(-1,-1)}^{a}(u, v)=J(u) \geq \frac{1}{\sqrt{|K(1,1)|}}=$ $J_{(-1,-1)}^{a}\left(u_{1}, v_{1}\right)$. So $\left(u_{1}, v_{1}\right)$ is the solution described by Theorem 1.1.

\section{Proof of proposition 3.1.}

property $i)$. First we express $I(u, v)$ as

$$
I(u, v)=\int_{\mathbf{R}^{3}} u^{2} v^{2}+\left(a^{2}-\omega^{2}\right) \int_{\mathbf{R}^{3}} v^{2}+2 a \int_{\mathbf{R}^{3}} u v^{2} .
$$

We have, using Hölder inequality and the Sobolev embedding (2),

$$
\begin{aligned}
\int_{\mathbf{R}^{3}} u^{2} v^{2} & \leq C\|u\|_{L^{6}}^{2}\|v\|_{L^{3}}^{2} ;\left|2 a \int_{\mathbf{R}^{3}} u v^{2}\right| \leq C\|u\|_{L^{6}}\|v\|_{L^{2}}^{3 / 2}\|v\|_{L^{6}}^{1 / 2} \leq \\
& \leq C\|u\|_{W^{1}}\|v\|_{L^{2}}^{3 / 2}\|v\|_{W^{1}}^{1 / 2} .
\end{aligned}
$$

This proves that $I(u, v)$ is well defined for $(u, v) \in W^{1} \times H^{1}$. Now, let us consider $(u, v) \in S_{\lambda, \mu}$. The relations (15) and (16) show that the following estimate holds :

$$
I(u, v) \geq\left(a^{2}-\omega^{2}\right) \int_{\mathbf{R}^{3}} v^{2}-C \lambda \mu^{1 / 2}\|v\|_{L^{2}}^{3 / 2}
$$

Thus $K(\lambda, \mu)>-\infty$. 
Now, let us consider a function $u \in H_{r}^{1}$ such that $u \equiv-a$ over $B(0,1)$. Let us denote $D^{c}$ the dilatation operator $D^{c}(u)(x)=u(c x)$. This operator satisfies $J\left(D^{c} u\right)=\frac{J(u)}{c}$ and we chose $c$ in order to achieve $J\left(D^{c} u\right)=\lambda$. We define a function $v \in H_{r}^{1}$ that satisfies $J(v)=\mu$ and $\operatorname{supp} v \subset\left\{x \in \mathbf{R}^{3} ; u(x)=a\right\}$. We compute for this couple of functions $I\left(D^{c} u, v\right)=-\omega^{2} \int_{\mathbf{R}^{3}} v^{2}<0$. Thus $K(\lambda, \mu)<0$.

property ii).Let $(\lambda, \mu, c)$ three positive reals. We prove first two preliminary properties.

Consider a minimizing sequence $\left(u_{n}, v_{n}\right) \in S_{\lambda, \mu}$ such that $K(\lambda, \mu)=$ $\lim _{n \rightarrow \infty} I\left(u_{n}, v_{n}\right)$. The sequence $\left(u_{n}, c^{1 / 2} v_{n}\right)$ satisfies :

$$
\left\{J\left(u_{n}\right)=\lambda ; J\left(c v_{n}\right)=c J\left(v_{n}\right) ; I\left(u_{n}, c^{1 / 2} v_{n}\right)=c I\left(u_{n}, v_{n}\right)\right\} .
$$

Thus we deduce

$$
K(\lambda, c \mu) \leq c K(\lambda, \mu)
$$

We consider a minimizing sequence $\left(u_{n}, v_{n}\right) \in S_{\mu, \mu}$ such that $I\left(u_{n}, v_{n}\right) \rightarrow K(\mu, \mu)$. The sequence $\left(D^{\frac{1}{c}} u_{n}, D^{\frac{1}{c}} v_{n}\right)$ satisfies

$$
\left\{J\left(D^{\frac{1}{c}} u_{n}\right)=c \mu ; J\left(D^{\frac{1}{c}} v_{n}\right)=c \mu ; I\left(D^{\frac{1}{c}} u_{n}, D^{\frac{1}{c}} v_{n}\right)=c^{3} I\left(u_{n}, v_{n}\right)\right\} .
$$

We have thus

$$
K(c \mu, c \mu) \leq c^{3} K(\mu, \mu) .
$$

We deduce from (18) and (19) the following :

$$
\begin{aligned}
K(1,1)= & K\left(\frac{\lambda}{\lambda}, \frac{\lambda}{\lambda}\right) \leq \frac{1}{\lambda^{3}} K(\lambda, \lambda)=\frac{1}{\lambda^{3}} K\left(\lambda, \mu \frac{\lambda}{\mu}\right) \\
& \leq \frac{\lambda}{\mu} \frac{1}{\lambda^{3}} K(\lambda, \mu)=\frac{1}{\mu \lambda^{2}} K(\lambda, \mu) \\
K(\lambda, \mu)= & K\left(\lambda, \frac{\mu}{\lambda} \lambda\right) \leq \frac{\mu}{\lambda} K(\lambda, \lambda) \leq \lambda^{3} \frac{\mu}{\lambda} K(1,1)=\lambda^{2} \mu K(1,1) .
\end{aligned}
$$

We deduce $K(\lambda, \mu)=\lambda^{2} \mu K(1,1)$. 
property iii). We prove in a first step

$$
K(\lambda, \mu)=\inf _{(u, v) \in S_{\lambda, \mu} \cap\left(C_{0}^{\infty}\right)^{2} \cap\{v \geq 0\} \cap\left\{0 \leq-a u \leq a^{2}\right\}} I(u, v)
$$

As $C_{0}^{\infty}$ is dense in $W^{1}$ and $H^{1}$, we have $K(\lambda, \mu)=$ $\inf _{(u, v) \in S_{\lambda, \mu} \cap\left[C_{0}^{\infty}\right]^{2}} I(u, v)$. Furthermore, let us denote $\left(u_{n}, v_{n}\right) \in S_{\lambda, \mu} \cap$ $C_{0}^{\infty}$ a minimizing sequence satisfying $K(\lambda, \mu)=\lim _{n \rightarrow \infty} I\left(u_{n}, v_{n}\right)$. We have $\left(u_{n},\left|v_{n}\right|\right) \in S_{\lambda, \mu}$, and $I\left(u_{n},\left|v_{n}\right|\right)=I\left(u_{n}, v_{n}\right)$. Thus we can suppose $v \geq 0$. We suppose also in the next $I\left(u_{n}, v_{n}\right)<$ 0 , according to property i). We denote the truncated function $u_{n}^{a}=$ $\left\{0, a u \geq 0 ; u,-a u \leq a^{2} ;-a,-a u \geq a^{2}\right\}$. This function satisfies $J\left(u_{n}^{a}\right) \leq$ $J\left(u_{n}\right)$ and $I\left(u_{n}^{a}, v_{n}\right) \leq I\left(u_{n}, v_{n}\right)$. We denote $\delta_{n}=\frac{J\left(u_{n}^{a}\right)}{J\left(u_{n}\right)} \leq 1$, and $\left(u_{n}^{\prime}, v_{n}^{\prime}\right)=T_{\left(\delta_{n}, \delta_{n}^{2}\right)}\left(u_{n}^{a}, v_{n}\right)$, where the operator $T$ is defined in (14). A direct computation shows that $\left(u_{n}^{\prime}, v_{n}^{\prime}\right) \in S_{\lambda, \mu} \cap\left(C_{0}^{\infty}\right)^{2} \cap\{v \geq 0\} \cap$ $\left\{0 \leq-a u \leq a^{2}\right\}$, and $I\left(u_{n}^{\prime}, v_{n}^{\prime}\right)=\frac{I\left(u_{n}^{a}, v_{n}\right)}{\delta_{n}^{2}} \leq I\left(u_{n}, v_{n}\right)$. So the minimum $K(\lambda, \mu)$ is also reached into the subset $S_{\lambda, \mu} \cap\left\{0 \leq-a u \leq a^{2}\right\}$, that ends the proof of $(20)$.

We suppose in this part without loss of generality $a>0$. In view of (20), we consider a couple of function $(u, v)$ such that

$$
(u, v) \in\left(C_{0}^{\infty}\right)^{2} \cap\{v \geq 0\} \cap\{-a \leq u \leq 0\},
$$

We recall now some notions of spherical reordering and we report for instance to [7], chapter I, for an introduction and a proof of the results used here. We introduce first some notations. Let $\Omega_{v}=\operatorname{supp} v, \Omega_{u}=\operatorname{supp} u$, and consider $\Omega$ an open bounded set such that $\Omega_{v} \cup \Omega_{v} \subset \Omega$. We denote $\Omega^{*}=[0$, meas $\{\Omega\}]$. For any measurable function $w: \Omega \rightarrow \mathbf{R}$, we introduce $\mu_{w}: \Omega^{*} \rightarrow \mathbf{R} \cup\{+\infty\} \cup\{-\infty\}$ the distribution function of $w$, as

$$
\mu_{w}(t)=\text { meas }\{x \in \Omega, w(x)<t\} .
$$

This is a increasing, right continuous function. We define $w^{*}$, the monodimensional increasing reordering of $w$, as

$$
\begin{aligned}
w^{*}(s) & =\inf \left\{t \in \mathbf{R}, \mu_{w}(t)>s\right\}, s \in \Omega^{*}, \\
w^{*}(\text { meas }(\Omega)) & =\operatorname{supessw.}
\end{aligned}
$$


Function $w^{*}$ is also increasing. The spherical increasing reordering of $w$ is defined as

$$
\bar{w}(x)=w^{*}\left(\alpha_{3} r(x)^{3}\right), x \in \widetilde{\Omega}=B(0, \text { meas }\{\Omega\}) .
$$

$\left(\alpha_{3}=\right.$ meas $\left.\{B(0,1)\}\right)$. We define also the monodimensional and spherical decreasing reordering of $w$ as

$$
w_{*}(s)=-(-w)^{*}(s) ; \underline{w}(x)=w_{*}\left(\alpha_{3} r(x)^{3}\right)
$$

For $(u, v)$ given by $(21)$, we deduce for instance the following property :

$$
u^{*}(0)=\inf u, u^{*}(\operatorname{meas}\{\Omega\})=0, v_{*}(0)=\sup v, v_{*}(\text { meas }\{\Omega\})=0 .
$$

We have the following inequality :

$$
J(\bar{u}) \leq J(u), J(v) \leq J(\underline{v}), I(\bar{u}, \underline{v}) \leq I(u, v)
$$

Where we have defined $(\bar{u}, \underline{v})(x)=0$, for $x \notin B(0$, meas $\{\Omega\})$. The first two inequality are direct applications of the Polya-Szego inequality. To prove the last one, we apply the Hardy Littlewood inequality to get

$$
\int_{\mathbf{R}^{3}}-(u+a)^{2} v^{2} d x \leq \int_{\mathbf{R}^{3}} \overline{(u+a)^{2}} \overline{\left(-v^{2}\right)} d x
$$

Consider the application $\phi_{a}(u)=\left\{0, u<-a ;(u+a)^{2}, u \geq-a\right\}$. As the increasing and decreasing reordering commute with an increasing application, we deduce from (21)

$$
\overline{(u+a)^{2}}=\overline{\phi_{a}(u)}=\phi_{a}(\bar{u})=(\bar{u}+a)^{2}
$$

In the same vein, we deduce

$$
\overline{\left(-v^{2}\right)}=-\underline{\left(v^{2}\right)}=-\underline{\phi_{0}(v)}=-\phi_{0}(\underline{v})=-\underline{v}^{2}
$$

We deduce from (23) $\int_{\mathbf{R}^{3}}(u+a)^{2} v^{2} d x \geq \int_{\mathbf{R}^{3}}(\bar{u}+a)^{2} \underline{v}^{2} d x$. Furthermore the reordering conserves the $L^{p}$ norms, so $\int_{\mathbf{R}^{3}} v^{2} d x=\int_{\mathbf{R}^{3}} \underline{v}^{2} d x$. These two results imply (22).

Let us consider a minimizing sequence $\left(u_{n}, v_{n}\right) \in S_{\lambda, \mu} \cap\left(C_{0}^{\infty}\right)^{2} \cap$ $\{v \geq 0\} \cap\{-a \leq u \leq 0\}$, such that $I\left(u_{n}, v_{n}\right)<0$. We denote $\delta_{n}^{u}=\frac{J\left(\overline{u_{n}}\right)}{J\left(u_{n}\right)}$, 
$\delta_{n}^{v}=\frac{J\left(v_{n}\right)}{J\left(v_{n}\right)}$, and we consider $\left(u_{n}^{\prime}, v_{n}^{\prime}\right)=T_{\left(\delta_{n}^{u} \delta_{n}^{v},\left(\delta_{n}^{u}\right)^{2}\right)}\left(\overline{u_{n}}, \underline{v_{n}}\right)$, where the operator $T$ is defined in (14). A direct computation shows that $\left(u_{n}^{\prime}, v_{n}^{\prime}\right) \in S_{\lambda, \mu}^{r} \cap\left(C_{0}^{\infty}\right)^{2} \cap\{v \geq 0\} \cap\{-a \leq u \leq 0\}$, and $I\left(u_{n}^{\prime}, v_{n}^{\prime}\right)=$ $\frac{I\left(\overline{u_{n}, v_{n}}\right)}{\left(\delta_{n}^{u}\right)^{2} \delta_{n}^{v}} \leq I\left(u_{n}, v_{n}\right)$, using (22), ending the proof of property iii).

Proof of lemma 3.1. We consider a radial minimizing subsequence achieving $\left(u_{\lambda}^{n}, v_{\mu}^{n}\right)_{n \geq 0}$ and we denote, up to a subsequence, $\left(u_{\lambda}^{n}, v_{\mu}^{n}\right) \rightarrow$ $\left(u_{\lambda}, v_{\mu}\right)$, as $n \rightarrow \infty$, weakly in $\left(W_{r}^{1}\right)^{2}$. We prove in a first step

$$
I\left(u_{\lambda}, v_{\mu}\right) \leq K(\lambda, \mu)
$$

For that aim, we estimate each term of $I\left(u_{\lambda}^{n}, v_{\mu}^{n}\right)$ given in (15). First, note that, according to (17) and Proposition 3.1, i), $\left(v_{\mu}^{n}\right)_{n \geq 0}$ is a bounded sequence in $L^{2}$. Thus it is also bounded in $H_{r}^{1}$. According to the Strauss compactness result, ([12]), $v_{\mu}^{n} \rightarrow v_{\mu}$ strongly in $L^{p}, 2<p<6$. We have also $u_{\lambda}^{n} \rightarrow u_{\lambda}$, weakly in $L^{6}$, according to (2). This implies

$$
\begin{aligned}
& \int_{\mathbf{R}^{3}}\left(u_{\lambda}^{n}\right)^{2}\left(v_{\lambda}^{n}\right)^{2}=\left\langle\left(u_{\lambda}^{n}\right)^{2},\left(v_{\lambda}^{n}\right)^{2}\right\rangle_{L^{3},\left(L^{3}\right)^{\prime} \approx L^{3 / 2}} \rightarrow \int_{\mathbf{R}^{3}} u_{\lambda}^{2} v_{\mu}^{2}, \text { as } n \rightarrow \infty . \\
& 2 a \int_{\mathbf{R}^{3}} u_{\lambda}^{n}\left(v_{\lambda}^{n}\right)^{2}=2 a\left\langle u_{\lambda}^{n},\left(v_{\lambda}^{n}\right)^{2}\right\rangle_{L^{6},\left(L^{6}\right)^{\prime} \approx L^{6 / 5}} \rightarrow 2 a \int_{\mathbf{R}^{3}} u_{\lambda} v_{\mu}^{2} \text { as } n \rightarrow \infty .
\end{aligned}
$$

These two limits, together with (11) and the Fatou's lemma allow to deduce

$\left(a^{2}-\omega^{2}\right) \int_{\mathbf{R}^{3}} v_{\lambda}^{2} \leq\left(a^{2}-\omega^{2}\right) \lim _{n \rightarrow \infty} \int_{\mathbf{R}^{3}}\left(v_{\lambda}^{n}\right)^{2}$. Blending all these results, we get

$$
K(\lambda, \mu) \geq \int_{\mathbf{R}^{3}} u_{\lambda} v_{\mu}+2 a \int_{\mathbf{R}^{3}} u_{\lambda} v_{\mu}+\left(a^{2}-\omega^{2}\right) \int_{\mathbf{R}^{3}} v_{\mu}
$$

that proves (24).

We denote $\delta^{u}=\frac{J\left(u_{\lambda}\right)}{\lambda} \leq 1, \delta^{v}=\frac{J\left(v_{\mu}\right)}{\mu} \leq 1$. First notice that if $\delta^{u}=0$ (resp. $\delta^{v}=0$ ) then we deduce from $(25) K(\lambda, \mu) \geq\left(a^{2}-\omega^{2}\right) \int_{\mathbf{R}^{3}}\left(v_{\mu}\right)^{2}$ (resp. $K(\lambda, \mu) \geq 0$ ), contradicting Proposition 3.1,i). Thus we can consider $\left(u_{\lambda}^{\prime}, v_{\mu}^{\prime}\right)=T_{\left(\delta^{u} \delta^{v},\left(\delta^{u}\right)^{2}\right)}\left(u_{\lambda}, v_{\mu}\right)$. A direct computation shows 
that $\left(u_{n}^{\prime}, v_{n}^{\prime}\right) \in S_{\lambda, \mu}^{r}$. We deduce, according to (24),

$$
K(\lambda, \mu) \leq I\left(u_{\lambda}^{\prime}, v_{\mu}^{\prime}\right)=\frac{I\left(u_{n}, v_{n}\right)}{\left(\delta^{u}\right)^{2} \delta^{v}} \leq \frac{K(\lambda, \mu)}{\left(\delta^{u}\right)^{2} \delta^{v}}<0 .
$$

Thus $\delta^{u}=1$ and $\delta^{v}=1$ and we have proved the strong convergence of a subsequence in $\left(W_{r}^{1}\right)^{2}$. We deduce from $(24) K(\lambda, \mu)=I\left(u_{\lambda}, v_{\mu}\right)$. A straightforward consequence of this equality in the proof of (24) is $\left(a^{2}-\omega^{2}\right) \int_{\mathbf{R}^{3}} v_{\mu}^{2}=\left(a^{2}-\omega^{2}\right) \lim _{n \rightarrow \infty} \int_{\mathbf{R}^{3}}\left(v_{\lambda}^{n}\right)^{2}$. So, up to a subsequence, $\left(v_{\mu}^{n}\right)_{n \geq 0}$ is also a strongly convergence in $L^{2}$, proving the strongly convergence of $\left(v_{\mu}^{n}\right)_{n \geq 0}$ in $H_{r}^{1}$.

Remark 3.2. We point out that, if $(u, v)$ is a finite energy solution of $R_{a}(-1,-1)$ in the case $0<\omega^{2}<a^{2}$, then $v$ has an exponential decay at infinity (this can be viewed as a consequence of the work of Kato [10]).

\section{Existence results via comparison and O.D.E. arguments}

We prove in this section the theorems 1.2 and 1.3 considering the equivalent systems

$$
R^{r}\left(\varepsilon_{1}, \varepsilon_{2}\right):\left\{\begin{array}{l}
-\partial_{r}\left(r^{2} \partial_{r} u\right)=r^{2}\left(\varepsilon_{1} u v^{2}+\omega_{1}^{2} u\right) \\
-\partial_{r}\left(r^{2} \partial_{r} v\right)=r^{2}\left(\varepsilon_{2} v u^{2}+\omega_{2}^{2} v\right)
\end{array}\right.
$$

that are satisfied by any radial solution of $R\left(\varepsilon_{1}, \varepsilon_{2}\right)$ expressed in polar coordinates. These systems are considered with initial conditions

$$
\left\{\begin{array}{l}
\partial_{r} u(0)=0 ; u(0)=a \\
\partial_{r} v(0)=0 ; v(0)=b
\end{array} .\right.
$$

Thus the associated solutions of $R\left(\varepsilon_{1}, \varepsilon_{2}\right)$ are smooth in a neighborhood of the origin. For a function, let say $u(r)$, a capital letter denotes the function $U(r)=r u(r)$.

Proof of Theorem 1.2. It is a straightforward computation to show that the system 


$$
\left\{\begin{array}{l}
-U^{\prime \prime}-\omega_{1}^{2} U-\frac{U V^{2}}{r^{2}}=0 \\
-V^{\prime \prime}-\omega_{2}^{2} V-\frac{V U^{2}}{r^{2}}=0
\end{array}\right.
$$

is equivalent to $R^{r}(+1,+1),(27)$ for initial conditions $U^{\prime}(0)=a, V^{\prime}(0)=$ $b, U(0)=V(0)=0$. We remark that this system admits a Lyapunov functional defined by

$$
f=\left(U^{\prime}\right)^{2}+\omega_{1}^{2} U^{2}+\left(V^{\prime}\right)^{2}+\omega_{2}^{2} V^{2}+\frac{U^{2} V^{2}}{r^{2}} .
$$

It is a positive function satisfying $f^{\prime}(r)=-2 \frac{U^{2} V^{2}}{r^{3}} \leq 0$. This implies that $(u, v)=\frac{1}{r}(U, V)$ is a global solution of $R^{r}(+1,+1)$ that satisfies $\left(\omega_{1}^{2} u^{2}+\omega_{2}^{2} v^{2}\right)(r) \leq \frac{f(0)}{r^{2}}$.

\section{Proof of Theorem 1.3.}

We show this result considering the equivalent equation of $R(-1)$ expressed in polar coordinate :

$$
-\partial_{r}\left(r^{2} \partial_{r} u\right)=r^{2}\left(-u^{3}+\omega^{2} u\right), \omega \in \mathbf{R} .
$$

This is a particular case of $R^{r}(-1,-1)$. We consider this equation associated to initial conditions $\left\{u(0)=a, \partial_{r} u(0)=0\right\}$.

We begin this proof with some preliminary remarks and notations. For a continuous function $q, \lambda_{n}(\Omega)$ denotes in this section the $n$-th eigenvalues of the Laplacian with Dirichlet boundary conditions considered on a bounded regular set $\Omega$. We denote also $\phi_{1, \Omega}$ the positive first eigenvalue associated to $\lambda_{1}(\Omega):-\Delta \phi_{1}=\lambda_{1} \phi_{1}, \phi_{1}>0$ in $\Omega, \phi_{1}=0$ on $\partial \Omega,\left\|\phi_{1, \Omega}\right\|_{L^{\infty}}=1$. We recall some well known facts about the equation (see [1] for instance)

$$
-\Delta u+u^{3}=\omega^{2} u \text { in } \Omega, u=0 \text { on } \partial \Omega .
$$

It does not exists any non trivial solution $u$ of (29) for $\omega^{2} \leq$ $\lambda_{1}(\Omega)$. The functions $\varepsilon \phi_{1, \Omega}, \varepsilon \geq 0$ satisfies $-\Delta \varepsilon u_{m}-\omega^{2} u_{m}+\varepsilon^{3} u_{m}^{3}=$ $\left(\lambda_{1}(\Omega)-\omega^{2}\right) \varepsilon \phi_{1, \Omega}+\varepsilon^{3} \phi_{1, \Omega}^{3}$. This means that $\varepsilon \phi_{1, \Omega}$ is a supersolution of (29) if $\varepsilon^{2} \leq \omega^{2}-\lambda_{1}(\Omega)$. The functions $u \equiv M \geq 0$ satisfy $-\Delta u+u^{3} \omega^{2} u=$ $M^{3}-\omega^{2} M$. Thus $M$ is a supersolution of (29) if $|\omega| \leq M$. We deduce 
the existence of a unique maximal non trivial positive solution $u$ of (29) that satisfies

$$
\left(\omega^{2}-\lambda_{1}(\Omega)\right)^{1 / 2} \phi_{1, \Omega} \leq u \leq|\omega| \text {, if } \omega^{2}>\lambda_{1}(\Omega) .
$$

We make also some preliminary remarks about the solutions of (28). Let $u$ a solution of (28) with initial conditions $\left\{u(0)=a, \partial_{r} u(0)=0\right\}$. We consider the function $E(u)=\left(\partial_{r} u\right)^{2}+\omega^{2} u^{2}-\frac{u^{4}}{2}$. Computing $\partial_{r}\left(r^{p} E(u)(r)\right)$ for $p \geq 0$ an integer and integrating, we find

$$
r^{p} E(u)(r)=p \int_{0}^{r} r^{p-1}\left(\omega^{2} u^{2}-\frac{u^{4}}{2}\right) d r+(p-4) \int_{0}^{r} r^{p-1}\left(\partial_{r} u\right)^{2} d r
$$

This is an analogous of the Pohozaev relation if one takes $p=3$. We state also the radial versions of the energy relation : we multiply (28) with $u$ and integrate over $[0, r]$ to get

$$
-\frac{1}{2} r^{2} \partial_{r}\left(u^{2}\right)(r)=\int_{0}^{r} r^{2}\left(\omega^{2} u^{2}-u^{4}\right) d r-\int_{0}^{r} r^{2}\left(\partial_{r} u\right)^{2} d r
$$

Also, let us denote $v$ a positive solution of (28) on a segment $[\alpha, \alpha+\beta], \alpha \geq 0, \beta>0$. We consider on the intervall $[\alpha+\delta, \alpha+\beta+\delta]$ the functions $v_{\gamma, \delta}(r)=\gamma v(r-\delta) \frac{(r-\delta)}{r}$, with $\alpha-\delta>0, \gamma \geq 0$. Remark that $r v_{\gamma, \delta}(r)=V_{\gamma, \delta}(r)=\gamma V(r-\delta)$. We denote $\Delta_{r}=\partial_{r}^{2}+\frac{2}{r} \partial_{r}$ the Laplacian expressed in polar coordinate and compute

$$
\begin{aligned}
r\left(-\Delta_{r} v_{\gamma, \delta}+v_{\gamma, \delta}^{3}-\omega^{2} v_{\gamma, \delta}\right)= & -V_{\gamma, \delta}^{\prime \prime}+\frac{V_{\gamma, \delta}^{3}}{r^{2}}-\omega^{2} V_{\gamma, \delta}=-\gamma V^{\prime \prime}(r-\delta) \\
& +\gamma^{3} \frac{V^{3}(r-\delta)}{r^{2}}-\omega^{2} \gamma V(r-\delta) \\
= & \gamma^{3} \frac{V^{3}(r-\delta)}{r^{2}}-\gamma \frac{V^{3}(r-\delta)}{(r-\delta)^{2}} .
\end{aligned}
$$

Thus $\operatorname{sgn}\left(-\Delta_{r} v_{\gamma, \delta}+v_{\gamma, \delta}^{3}-\omega^{2} v_{\gamma, \delta}\right)=\operatorname{sgn}\left(\gamma-\frac{r}{r-\delta}\right)$. This means that in $[\alpha+\delta, \alpha+\delta+\beta]$,

$$
\begin{aligned}
v_{\gamma_{1}, \delta} \text { is a subsolution if } \gamma_{1} & \leq \inf _{r \in[\alpha+\delta, \alpha+\beta+\delta]} \frac{r}{(r-\delta)} \\
v_{\gamma_{2}, \delta} \text { is a supersolution if } \gamma_{2} & \geq \sup _{r \in[\alpha+\delta, \alpha+\delta+\beta]} \frac{r}{(r-\delta)}
\end{aligned}
$$


Existence of a global solution to (28): we consider without loss of generality $a>0$, the equation being invariant by the transformation $u \rightarrow-u$. Let us denote $u_{\alpha}$, for $\omega^{2}>\lambda_{1}(B(0, \alpha))=\left(\frac{\pi}{\alpha}\right)^{2}$, the unique non trivial radial solution of $(29)$ on the ball $B(0, \alpha)$. It is easy to check using (30), (see for example [1]) that $\sup _{x \in B(0, \alpha)} u_{\alpha}(x)=u_{\alpha}(0)$, that $\alpha \rightarrow f(\alpha)=u_{\alpha}(0)$ is an increasing continuous function of $\alpha \in\left(\frac{\pi}{|\omega|}, \infty\right)$ and $\lim _{\alpha \rightarrow \frac{\pi}{|\omega|}} f(\alpha)=0, \lim _{\alpha \rightarrow \infty} f(\alpha)=|\omega|$. Thus we can denote $f^{-1}(a)$ the inverse of $f$, which is defined for $0<a<|\omega|$. So, for every $0<a<$ $|\omega|$, there exists an unique solution $u_{s, f^{-1}(a)}$ of (28) with initial conditions $\left\{u_{s}^{\prime}(0)=0, u_{s}(0)=a\right\}$ over $\left[0, f^{-1}(a)\right]$, which is nothing else but $u_{f^{-1}(a)} \in H_{0}^{1}\left(B\left(0, f^{-1}(a)\right)\right)$ expressed in polar coordinates.

We denote in the sequel for short $u=u_{s, f^{-1}(a)}$. We know from the above construction that $u$ have one zero $\zeta_{1}(u)=f^{-1}(a)$. Let us suppose that $u$ possesses $N$ zeros $\left(\zeta_{i}(u)\right)_{1<i<N^{*}}$, and consider the evolution system (28) with data given at $r=\zeta_{N}(u)$, i.e. with initial conditions $\left(0, \partial_{r} u\left(\zeta_{N}(u)\right)\right)$. We claim that $u$ satisfies (with the convention $\left.\zeta_{0}(u)=0\right)$,

$$
\frac{\pi}{|\omega|}<\zeta_{N+1}(u)-\zeta_{N}(u)<\zeta_{N}(u)-\zeta_{N-1}(u)
$$

Notice that if $u$ is a nontrivial solution over $\left[0, \zeta_{N}(u)\right]$, then $|u| \in$ $H_{0}^{1}\left(\left[\zeta_{i}(u), \zeta_{i+1}(u)\right]\right), 1 \leq i \leq N$ is a non trivial positive solution. So we have $\lambda_{1}\left(\left[\zeta_{i}(u), \zeta_{i+1}(u)\right]\right)^{2}=\left(\frac{\pi}{\zeta_{i+1}(u)-\zeta_{i}(u)}\right)^{2}<\omega^{2}$, that proves the left hand side of (35).

We denote in the sequel $I_{N}=\left[\zeta_{N}(u), 2 \zeta_{N}(u)-\zeta_{N-1}(u)\right]$ and $u_{I_{N}}$ the unique non trivial positive solution of (28) that belongs to $H_{0}^{1}\left(I_{N}\right)$. According to (33), we consider $u_{\gamma, \delta}$ the subsolution of $u_{I_{N}} \in H_{0}^{1}\left(I_{N}\right)$, with $\delta=\zeta_{N}(u)-\zeta_{N-1}(u)$, and $\gamma=\inf _{r \in I_{N}} \frac{r}{(r-\delta)}=\frac{2 \zeta_{N}(u)-\zeta_{N-1}(u)}{\zeta_{N}(u)}$. So we have

$$
\left(\partial_{r} u_{I_{N}}\right)^{2}\left(\zeta_{N}(u)\right) \geq\left(\partial_{r} u_{\gamma, \delta}\right)^{2}\left(\zeta_{N}(u)\right)
$$

Furthermore, $(31$, case $\mathrm{p}=0)$ proves that

$$
E\left(u_{\gamma, \delta}\right)\left(\zeta_{N}(u)\right)=\left(\partial_{r} u_{\gamma, \delta}\right)^{2}\left(\zeta_{N}(u)\right)>\left(\partial_{r} u_{\gamma, \delta}\right)^{2}\left(2 \zeta_{N}(u)-\zeta_{N-1}(u)\right) .
$$

We have

$$
\left(\partial_{r} u_{\gamma, \delta}\right)^{2}\left(2 \zeta_{N}(u)-\zeta_{N-1}(u)\right)=\left(\partial_{r} u\right)^{2}\left(\zeta_{N}(u)\right),
$$


thus $\left(\partial_{r} u_{\gamma, \delta}\right)^{2}\left(\zeta_{N}(u)\right)>\left(\partial_{r} u\right)^{2}\left(\zeta_{N}(u)\right)$. This inequality together with (36) implies

$$
\left(\partial_{r} u_{I_{N}}\right)^{2}\left(\zeta_{N}(u)\right)>\left(\partial_{r} u\right)^{2}\left(\zeta_{N}(u)\right) .
$$

Now, let us consider $g=\inf \left(|u|, u_{I_{n}}\right)$. This function has a zero at $\zeta_{N}(u)$. We denote $\zeta_{N+1}(g) \leq 2 \zeta_{N}(u)-\zeta_{N-1}(u)$ its next zero, and $I_{g}=\left[\zeta_{N}(u), \zeta_{N+1}(g)\right]$. According to $(37), g$ satisfies $g(r)=|u|(r)$ on a positive neighborhood of $\zeta_{N}(u)$. Furthermore, $g$ is a supersolution of (28) over $I_{g}$. This means that there exists a positive solution $H_{0}^{1}\left(I_{g}\right) \ni u^{\prime} \leq g$ of (28). Now, let us suppose that $\zeta_{N+1}(g)=$ $2 \zeta_{N}(u)-\zeta_{N-1}(u)$. This implies that there exists two distinct positive solutions $u^{\prime}$ and $u_{I_{n}}$ that belongs to $H_{0}^{1}\left(I_{g}\right)$, absurd. Thus we must have $\zeta_{N+1}(g)<2 \zeta_{N}(u)-\zeta_{N-1}(u)$. Now, as $|u|$ is the unique positive solution of (28) that belongs to $H_{0}^{1}\left(I_{g}\right)$, we deduce $|u|=u^{\prime} \leq \inf \left(|u|, u_{I_{n}}\right)$. Thus $|u|=\inf \left(|u|, u_{I_{n}}\right)$. We get $\zeta_{N+1}(u)<2 \zeta_{N}(u)-\zeta_{N-1}(u)$, ending the proof of (35).

Using (35), we are able to construct by an induction argument a global solution of $(28)$ for every initial conditions $\left\{u_{s}^{\prime}(0)=0, u_{s}(0)=a\right\}$ satisfying $\omega^{2}>a^{2}$.

\section{Asymptotic behavior:}

As $|u|$ is the unique positive solution that belongs to $H_{0}^{1}\left(\left[\zeta_{i}(u), \zeta_{i+1}(u)\right]\right), 1 \leq i$, it satisfies the estimation $u^{2}<\omega^{2}$. Furthermore the function $U(r)=r u(r)$ satisfies the equation $\left\{-U^{\prime \prime}-\omega^{2} U+\frac{U^{3}}{r^{2}}=0\right\}$ considered with initial condition $\left\{U^{\prime}(0)=a, U(0)=0\right\}$. The functional $f=\left(U^{\prime}\right)^{2}+\omega^{2} U^{2}-\frac{U^{4}}{2 r^{2}}$, satisfies $f^{\prime}(r)=2 \frac{U^{4}(r)}{r^{3}} \geq 0$. Furthermore we compute $r^{2} E(u)(r)=f(r)+$ $r \partial_{r}\left(u^{2}\right)+u^{2}$. Evaluating this last expression at the sequence of points $\left\{\zeta_{i}\left(\partial_{r} u\right)\right\}_{i \geq 0}$, we get $\limsup _{r \rightarrow \infty} r^{2}\left(\omega^{2} u^{2}-\frac{u^{4}}{2}\right)(r) \geq f(0)>0$, that proves the first limit. From $(31$, case $\mathrm{p}=0)$ and the proof above, $E(u)(r)$ is a positive decreasing function. Thus $\lim _{r \rightarrow \infty} E(u)(r)=$ $E(u)(\infty)$, and $\lim _{r \rightarrow \infty} \partial_{r} u(r)=0$. Thus $\lim _{r \rightarrow \infty} u(r)=u(\infty)$ is a particular solution of $(28)$ that satisfies $u(\infty)^{2}<\omega^{2}$. So $u(\infty)=0$, that proves the second limit and ends the proof of the theorem.

Acknowledgments. The author is grateful to P. Caldiroli, M. Esteban and C. Stuart for stimulating conversations and useful references. 


\section{References}

[1] H. Berestycki, Les nombres de solutions de certains problèmes semilineéaires elliptiques, J. Funct. Anal., 40, 1-29, (1981).

[2] B. Hanouzet, Espaces de Sobolev avec poids et application au problème de Dirichlet dans un demiespace, Comm. Math. Phys, XLVI, 227-272, (1971).

[3] E. Gunzig and P. Nardone, Gravitational instability of empty Minkowski space-time, Physics letters, 118B, 324-327, (1982).

[4] P.L.H. Berestycki, Non linear scalar field equation i and ii, Arch. Rat. Mech. Anal., 82, 313-375, (1982).

[5] T. Jayant and V. Narlikar, Gravity, Gauge theory and quantum cosmology, D. Reidel Publishing Company, 1986.

[6] J.-M. Mercier, Global existence and long time estimation for an integrodifferential system, Preprint dell' Universita di Pisa 2.275.1047, to be published in Richerche di Matematica, 1997.

[7] J. Mossino, Inegalites isoperimetriques et applications a la physique, Hermann, Paris, 1984.

[8] P.H. Rabinowitz, Minimax methods in critical point theory with applications to differential equations, Conference board of the mathematical sciences, 65, (1984).

[9] M. Struwe, Variational methods. Applications to nonlinear partial differential equations and Hamiltonian systems, Springer Verlag, 1990.

[10] T. Kato, Growth properties of solutions of the reduces wave equation with a variable coefficient, Comm. in Pure and Applied Math, 12, 403-425, (1959).

[11] P.R.V. Benci, Critical point theorems for indefinite functionals, Inventiones math, 52, 241-273, (1979).

[12] W.A. Strauss, Existence of solitary waves in higher dimensions, Comm. Math. Phys, 55, 149-162, (1977).

École Polytechnique

Centre de Mathématiques Appliquées

U.A. CNRS 756

91128 PALAISEAU CEDEX (France)

E-mail address: jeanmarc.mercier@free.fr

Recibido: 27 de Abril de 1999

Revisado: 31 de Enero de 2000 